\title{
Fast identification of dislocations in semiconductor materials by electron channeling contrast imaging using a scanning electron microscope
}

\author{
Luyang $\operatorname{Han}^{1}$ and Yongkai Zhou ${ }^{2}$ \\ 1. Carl Zeiss Microscopy GmbH, Carl-Zeiss-Strasse 22, 73447 Oberkochen, Germany \\ 2. Carl Zeiss Microscopy GmbH, 4 Engineering Drive 3, 117576, Singapore
}

Lattice defects such as dislocations have a critical influence on the performance of semiconductor material. Traditionally the dislocation lines are analyzed by transmission electron microscopy (TEM). In this contribution we present an alternative technique to identify dislocation as well as their types using electron channeling contrast imaging (ECCI) in a scanning electron microscope (SEM). Compared to TEM a large amount of dislocation lines can be identified in very short time without modification of the sample. This technique can also be combined with TEM lamella preparation, where a defined dislocation can be localized and extracted using a FIB-SEM system.

To demonstrate the procedure an epitaxial GaN thin film grown on sapphire substrate is used. The GaN thin film is grown by molecular chemical vapor deposition (MOCVD) along the [0001] direction of sapphire, producing various kinds of dislocations [1]. The sample is investigated using ZEISS GeminiSEM 500 FE-SEM with a PIN-diode BSE detector under the objective lens. By adjusting the strength of the scanning coil it is possible to move the pivot point of scanning electron down to the surface of the sample. The electron beam will then rock on the sample with different incident angles, forming the selected area channelling pattern (SACP).

The channelling contrast depends on the convergence angle of the electron beam as well as the positioning of the detector with respect to the sample. To optimize the condition for ECCI the SACP is recorded at different working distance (WD), corresponding to different angular detection ranges of the BSE detector. The grey level variation along the [11-20] band is extracted and shown in Figure 1. The relative signal change along this direction is chosen as the criterion for the strength of channelling contrast. In general, smaller WDs or equivalently smaller take-off angles lead to better channelling contrast.

By checking the SACP the sample can be oriented in a specific direction so that Bragg conditions for a certain lattice plane are fulfilled. ECCI images of dislocations for different orientations are shown in Figure 2. The signal variation around the dislocation cores with respect to sample orientation carries information about the dislocation type [2]. When imaged along [0001] direction, the dislocations show no preferred contrast variation, while imaged under [20-20] and [11-20] diffraction conditions, most of the dislocations show 2 types of contrast variation, which are exactly $180^{\circ}$ rotated with respect to each other. This distinct feature is related to the handedness of screw dislocations in GaN.

\section{References:}

[1] Fong Kwong Yam et al, Optoelectronics - Materials and Techniques ed. P. Predeep (InTech) p. 99.

[2] G. Naresh-Kumar et al, Physical Review Letters, 108 (2012), p.135503. 

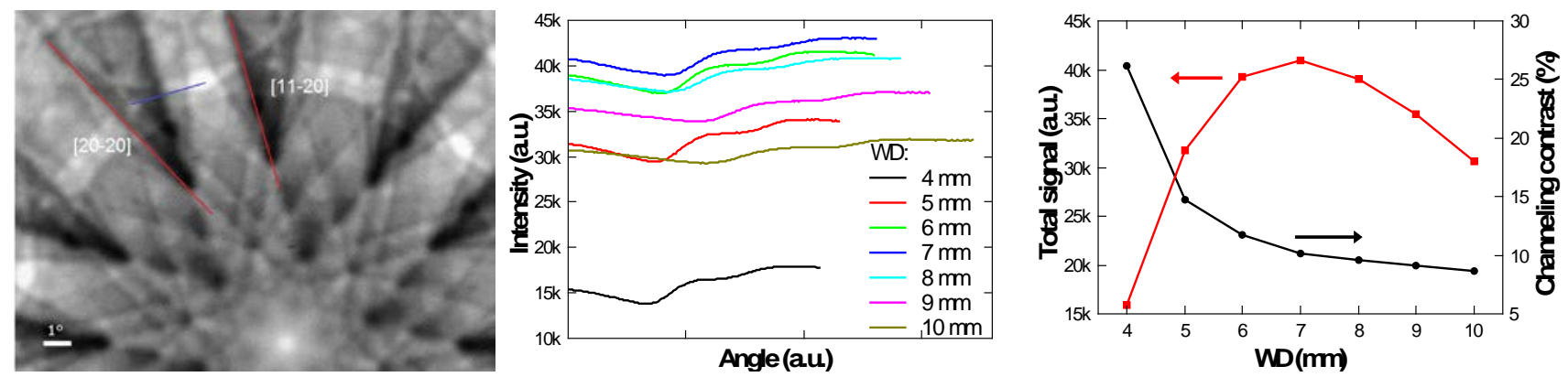

Figure 1. The left image shows the selected area channeling contrast on a GaN thin film with $20 \mathrm{kV}$ electron energy at $4 \mathrm{~mm}$ WD. The contrast profile across the [11-20] band (blue line) with different WD is shown in the middle plot. The average back-scattered signal level (red squares) as well as relative contrast of channeling (black dots) are summarized in the right plot.
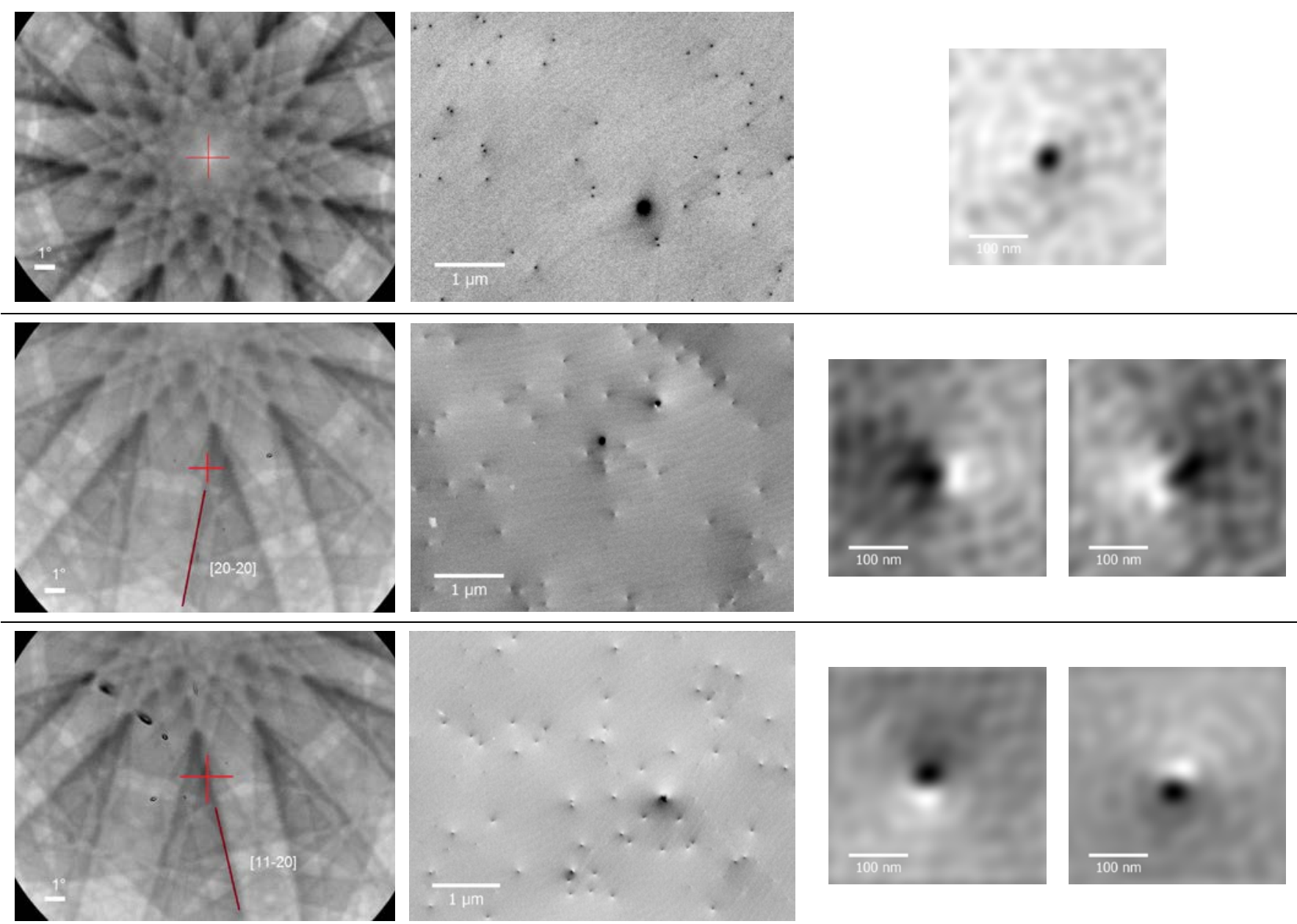

Figure 2. ECCI captured at three different beam conditions: along [0001] axis (first row), fulfilling [2020] (second row) and [11-20] (third row) diffraction conditions. The corresponding SACP are shown in the left column. The red crosses indicate the orientation of the electron beam in ECCI mode. Center column shows the ECCI image of the dislocations. The right column shows the enlarged images of the dislocation cores, where the grey level variation around the dislocation core carries information about the specific dislocation type. 